\title{
Anesthesiologic management of pregnant women with SARS-COV-2 infection undergoing cesarean delivery
}

Background: Pregnant women are usually more susceptible to infection due to typical physiological and mechanical changes, such as increased heart rate, stroke volume and pulmonary residual capacity. The aim of this study was to evaluate an innovative anesthesiologic opioid-free management protocol in symptomatic pregnant women, with COVID-19 and with oxygen therapy, undergoing cesarean delivery with spinal anesthesia. Methods: With the patient in the sitting position, spinal anesthesia was performed at the $\mathrm{L} 1-\mathrm{L} 2$ level. Vertebral level has been identified starting from the sacrum, we counted the laminae in the caudal-to-cephalad direction, which was then marked with a surgical pen. The technique was performed in asepsis, in the subarachnoid space after vision of clear CephaloSpinal Fluid (CSF) in the spinal needle 27 Gauge, without letting out the CSF, bupivacaine $0.5 \% 10 \mathrm{mg}$, dexmedetomidine $10 \mu \mathrm{g}$ and dexamethasone $4 \mathrm{mg}$ was injected. Results: During the study period, 40 pregnant women with one or more symptoms and supplemental oxygen ( $\left.\mathrm{FiO}_{2} 35-40 \%\right)$ who underwent cesarean delivery were included in the study. All pregnant women had pain visual analog scale $(\mathrm{VAS}) \leq 3$, and no pregnant women required rescue dose. Adverse effects, such as nausea, vomiting, shivering, or pruritus were not recorded in any case. After a mean of 2.5 hours from the spinal anesthesia, all the included women had a complete motility of the lower limbs and were able to mobilize independently within 12 hours after delivery. Mean time to first flatus was about 8 hours after delivery. Conclusions: Pregnant women in COVID-19 can safely receive intrathecal dexamethasone and dexmedetomidine during planned cesarean delivery.

\section{Keywords}

Pregnancy; Anesthesia; Delivery; COVID-19; SARS-COV-2; 2019-nCOV; Cesarean delivery; Spinal anesthesia; Neuraxial anesthesia; Labour; Fetus; Pneumonia

\section{Introduction}

In December 2019 a novel Coronavirus, SARS-COV-2, was identified as the cause of a severe acute respiratory syndrome, label COVID-19 [1]. COVID-19 could range from asymptomatic infection to severe viral pneumonia with respiratory failure.

Pregnant women are usually more susceptible to infection due to typical physiological and mechanical changes, such as increased heart rate, stroke volume and pulmonary residual capacity [2]. Fever, cough, and dyspnea are the most common symptoms of COVID-19 in pregnant women [3-5]. They are more predisposed to superimposed bacterial infections due to direct mucosal injury and dysregulation of immune responses after viral pneumonia [6-8]. The evidence of physiological and mechanical changes during pregnancy may influence anesthetic management in women undergoing cesarean delivery while positive at SARS-COV-2.

The aim of this study was to evaluate an innovative anesthesiologic opioid-free management protocol in pregnant women with SARS-COV-2 undergoing cesarean delivery.

\section{Materials and methods 2.1 Study design}

This was a cohort study of pregnant women with SARSCOV-2 infection referred to University of Naples Federico II (Naples, Italy) from 1 September 2020 to 15 December 2020.

Inclusion criteria were pregnant women with confirmed COVID-19 undergoing cesarean delivery. A confirmed case of COVID-19 was defined as a positive result on real-time reverse-transcriptase-polymerase-chain-reaction (RT-PCR) assay of nasal and pharyngeal swab specimens. Only women at or after 34 weeks of gestation, and only those with one or more symptoms (including fever, cough, fatigue, and shortness of breath) of COVID-19 and with oxygen therapy were included in the study.

\subsection{Anesthesiologic protocol}

In our center, a path dedicated to COVID-19 patients was set up, including a dedicated delivery room, an operating room and a hospital ward. Each room was equipped with a clean path with a room used for dressing the operators and a dirty path with a room used for undressing and sanitizing the operators. All the providers used the following PPE: waterproof suit, double boots, double gloves, protective goggles, FFP3 mask, visor. Patients were asked to wear surgical masks. 
In the operating room, a venous access was placed (18-16 $\mathrm{G})$ and antibiotic prophylaxis was administered (Cefazolin 1 or $2 \mathrm{~g}$ iv) 30 minutes before skin incision. Pantorc $40 \mathrm{mg}$ iv was also used. Vital signs were monitored: SpO2, ECG, NIBP each 2.5 minutes until fetal extraction, then every 5 minutes. A co-loading was started with $500 \mathrm{~mL}$ of crystalloids iv. We proceed to an intraoperative fluid administration of $15-20 \mathrm{~mL} / \mathrm{kg} /$ hour of iv crystalloids. With the patient in the sitting position, spinal anesthesia was performed at the L1-L2 level. Vertebral level has been identified starting from the sacrum, we counted the laminae in the caudalto-cephalad direction, which was then marked with a surgical pen. The technique was performed in asepsis, in the subarachnoid space after vision of clear Cephalo-Spinal Fluid (CSF) in the spinal needle 27 Gauge, without letting out the CSF, bupivacaine $0.5 \% 10 \mathrm{mg}$, dexmedetomidine $10 \mu \mathrm{g}$ and dexamethasone $4 \mathrm{mg}$ was injected. The patient was placed supine with a wedge under the left side. In case of maternal hypotension (MAP $<60 \mathrm{mmHg}$, SAP $<90 \mathrm{mmHg}$ or $<20 \%$ of the initial values), if associated with maternal bradycardia, Ephedrine $0.1 \mathrm{mg} / \mathrm{kg}$ iv was administered, if associated with tachycardia, Phenylephrine $100 \mathrm{mcg}$ /each bolus ev was administered. At the time of fetal extraction, Oxytocin 5 IU iv was administered as a slow bolus ( 3 minutes for all patients, 5 minutes in case of cardiopathic patients). This was followed by $10 \mathrm{IU} /$ hour of Oxytocin in $500 \mathrm{~mL}$ in slow infusion in the following 2/4 hours post-partum. The infusion of Oxytocin started soon after birth. In case of poor uterine contraction or postpartum hemorrhage (defined as blood loss exceeding $1000 \mathrm{~mL}$ ), methylergometrine $0.4 \mathrm{mg}$ i.m. was administered. After surgery we administered intravenous paracetamol $1 \mathrm{~g}$ 3 times a day. Keterolac $30 \mathrm{mg}$ was available as rescue dose. Pain control was considered good in case of VAS $<4$.

The patients were contacted every 6 hours by videocall on the first postoperative day to establish: VAS, presence of adverse effects such as nausea or vomiting or pruritus, mobilization, time to first flatus and pharmacological therapy was based on the patients' responses.

\section{Results}

During the study period, 40 pregnant women with one or more symptoms and supplemental oxygen (FiO2 35-40\%) who underwent cesarean delivery were included in the study. Out of the 40 included women, 30 had planned cesarean delivery for prior cesarean delivery, 5 had cesarean delivery for breech presentation, 4 had cesarean delivery for placenta previa, and one had cesarean delivery for maternal request. No women were on anticoagulation at the time of delivery.

All pregnant women had VAS $\leq 3$, and no pregnant women required rescue dose. Adverse effects, such as nausea, vomiting, shivering, or pruritus were not recorded in any case. After a mean of 2.5 hours from the spinal anesthesia, all the included women had a complete motility of the lower limbs and were able to mobilize independently within 12 hours after delivery. Mean time to first flatus was about 8 hours after delivery.

\section{Discussion}

This study aimed to evaluate an innovative anesthesiologic management protocol in pregnant women with COVID-19 undergoing cesarean delivery. We opted for an opioid free management to avoid opioid-related side effects that may worse the symptoms of COVID-19. Opioids induce alterations in immune function at level of lymphocyte and phagocytic cell and increase susceptibility to infections [9].

We chose to inject dexamethasone $4 \mathrm{mg}$ due to a powerful anti-inflammatory action. Intrathecal dexamethasone administration may influence intraspinal prostaglandin synthesis by inhibiting cytosolic phospholipase A2 (cPLA2) in laminae I and II after peripheral tissue injury and the expression of cyclo-oxygenase 2 reducing the peripheral inflammatory state, postoperative pain and analgesic requirements [10-12]. Epidural dexamethasone plus levobupivacaine prolongs the duration of spinal analgesia during combined spinal-epidural for management of labor pain, with hemodynamic stability and limited maternal and neonatal adverse effects [13]. Some study revealed that epidural bupivacaine-dexamethasone admixture had the same analgesic potency as bupivacaine-fentanyl with opioid sparing and antiemetic effects [14].

In COVID-19 patients with established moderate-tosevere ARDS dexamethasone down-regulates pulmonary and systemic inflammation improving medical condition [15].

All of glucocorticoids have anti-inflammatory effect but we opted to administrate dexamethasone because it is $20-30$ times more potent than the natural hormone cortisol and 4-5 times than prednisone, moreover dexamethasone had a long half-life of 36-54 hours [15, 16].

Dexmedetomidine is a highly selective a-2 adrenergic agonist used like adjuvant in anesthesia. There were different possible mechanisms to explain the enhanced anesthetic efficiency. According to some researchers the action of $\alpha 2$-agonism of dexmedetomidine, induces vasoconstriction, which might contribute to prolonging the period of analgesia. Also dexmedetomidine potentiates the spinal block via a synergistic interaction between $\alpha 2$ receptors and sodium channels, resulting in a reduction in the dose of the local anesthetics required for achieving effective spinal anaesthesia for certain surgical procedures [17].

Dexmedetomidine $10 \mathrm{mcg}$ was administrated in spinal anesthesia in order to enhance the anesthetic effect in local anesthesia. Dexmedetomidine together with bupivacaine can increase the duration of anesthesia with shorter sensory and motor block onset compared with bupivacaine alone andit guarantee hemodynamic stability, consumption of rescue dose of analgesic drug reduce [18]. Intrathecal dexmedetomidine significantly reduced the occurrence of shivering during cesarean section, it inhibit the body's thermoregulatory center by the block of the transmission of body temperature information at the level of the spinal cord. It is important 
because shivering increase oxygen consumption and carbon dioxide production, with a certain impact on maternal physio logical function. Dexmedetomidine can reduce the use of opioids during cesarean delivery reducing the risk of nausea and vomiting. Also in the postoperative period it have an opioid sparing effect reducing rescue dose of opioid and analgesic [19]. Intrathecal dexmedetomidine during cesarean section appears safe both for the mother and for the fetus. Dexmedetomidine is not easily transferred through the placenta due to their fat-soluble characteristics so it does not increase neonatal adverse reactions during cesarean section. No significant differences in neonatal 1- and 5-min Apgar scores or cord blood gas parameters are observed. Also, it did not increase the incidence of adverse reactions such as maternal bradycardia and hypotension [20]. No abnormal symptoms or signs in the nervous system were found, which suggest that dexmedetomidine is a safe intrathecal adjuvant.

\section{Conclusions}

In summary, pregnant women in COVID-19 can safely receive intrathecal dexamethasone and dexmedetomidine during planned cesarean delivery.

\section{Author contributions}

CDA, GS, AC, CP, EG and MV conceived and designed the study; CDA, CP, EG and GiuS analyzed the data; MI, LG, GC, AnnM, GiuS and AlfM contributed reagents and materials; AC, AnnM, MV, GS, MI, LG, GC and AlfM wrote the paper.

\section{Ethics approval and consent to participate}

All subjects gave their informed consent for inclusion before they participated in the study. The study was conducted in accordance with the Declaration of Helsinki, and the approval for this study (Ethical Committee $\mathrm{N}^{\circ} 145 / 20$ ) was provided by the Ethical Committee of Federico II University of Naples, Italy, (Prof Domenico Del Forno) on 3 April 2020. IRB approval: 145/20 (University of Naples Federico II).

\section{Acknowledgment}

Thanks to all the peer reviewers for their opinions and suggestions.

\section{Funding}

This research received no external funding.

\section{Conflict of interest}

The authors declare no conflict of interest.

\section{References}

[1] Zhu N, Zhang D, Wang W, Li X, Yang B, Song J, et al. A novel coronavirus from patients with pneumonia in China, 2019. New England Journal of Medicine. 2020; 382: 727-733.

[2] WAPM (World Association of Perinatal Medicine). Working Group on COVID-19. Maternal and perinatal outcomes of pregnant women with SARS-COV-2 infection. Ultrasound in Obstetrics \& Gynecology. 2021; 57: 232-241.
[3] Boelig RC, Manuck T, Oliver EA, Di Mascio D, Saccone G, Bellussi F, et al. Labor and delivery guidance for COVID-19. American Journal of Obstetrics \& Gynecology MFM. 2020; 2: 100110.

[4] Boelig RC, Saccone G, Bellussi F, Berghella V. MFM guidance for COVID-19. American Journal of Obstetrics \& Gynecology MFM. 2020; 2: 100106.

[5] Api O, Sen C, Debska M, Saccone G, D'Antonio F, Volpe N, et al. Clinical management of coronavirus disease 2019 (COVID-19) in pregnancy: recommendations of WAPM-World Association of Perinatal Medicine. Journal of Perinatal Medicine. 2020; 48: 857866.

[6] Di Mascio D, Khalil A, Saccone G, Rizzo G, Buca D, Liberati M, et al. Outcome of coronavirus spectrum infections (SARS, MERS, COVID-19) during pregnancy: a systematic review and metaanalysis. American Journal of Obstetrics \& Gynecology MFM. 2020; 2: 100107.

[7] Silversides CK, Colman JM. Physiological changes in pregnancy. In Oakley C, Warnes CA (eds.) Heart disease in pregnancy (pp. 6-17). 2nd edn. Massachusetts, USA: Oxford. 2007.

[8] Jamieson DJ, Honein MA, Rasmussen SA, Williams JL, Swerdlow DL, Biggerstaff MS, et al. H1N1 2009 influenza virus infection during pregnancy in the USA. The Lancet. 2009; 374: 451-458.

[9] Alexander M, Daniel T, Chaudry IH, Schwacha MG. Opiate analgesics contribute to the development of post-injury immunosuppression. The Journal of Surgical Research. 2005; 129: 161-168.

[10] Sacerdote P. Opioid-induced immunosuppression. Current Opinion in Supportive and Palliative Care. 2008; 2: 14-18.

[11] Jo YY, Yoo JH, Kim HJ, Kil HK. The effect of epidural administration of dexamethasone on postoperative pain: a randomized controlled study in radical subtotal gastrectomy. Korean Journal of Anesthesiology. 2011; 61: 233-237.

[12] Min SH, Soh JS, Park JY, Choi SU, Lee HW, Lee JJ, et al. Epidural dexamethasone decreased inflammatory hyperalgesia and spinal $\mathrm{cPLA}_{2}$ expression in a rat formalin test. Yonsei Medical Journal. 2014; 55: 1631-1639.

[13] Wahdan AS, El-Sakka AI, Hassan AR, Mohamed MM, Gaafar HMI, Helmy NY. Epidural levobupivacaine versus a combination of levobupivacaine and dexamethasone in patients receiving epidural analgesia. Journal of Anaesthesiology Clinical Pharmacology. 2019; 35: 109-113.

[14] Khafagy HF, Refaat AI, El-Sabae HH, Youssif MA. Efficacy of epidural dexamethasone versus fentanyl on postoperative analgesia. Journal of Anesthesia. 2010; 24: 531-536.

[15] Villar J, Ferrando C, Martínez D, Ambrós A, Muñoz T, Soler JA, et al. Dexamethasone treatment for the acute respiratory distress syndrome: a multicentre, randomised controlled trial. The Lancet Respiratory Medicine. 2020; 8: 267-276.

[16] Alessi J, de Oliveira GB, Schaan BD, Telo GH. Dexamethasone in the era of COVID-19: friend or foe? An essay on the effects of dexamethasone and the potential risks of its inadvertent use in patients with diabetes. Diabetology \& Metabolic Syndrome. 2020; 12: 80.

[17] Xia F, Chang X, Zhang Y, Wang L, Xiao F. The effect of intrathecal dexmedetomidine on the dose requirement of hyperbaric bupivacaine in spinal anaesthesia for caesarean section: a prospective, double-blinded, randomized study. BMC Anesthesiology. 2018; 18: 74.

[18] Liu X, Zhang X, Wang X, Wang J, Wang H. Comparative evaluation of intrathecal bupivacaine alone and bupivacaine combined with dexmedetomidine in cesarean section using spinal anesthesia: a meta-analysis. Journal of International Medical Research. 2019; 47: 2785-2799.

[19] Wang YQ, Zhang XJ, Wang Y. Effect of intrathecal dexmedetomidine on cesarean section during spinal anesthesia: a meta-analysis of randomized trials. Drug Design, Development and Therapy. 2019; 13: 2933-2939.

[20] Sun S, Wang J, Wang J, Wang F, Xia H, Yao S. Fetal and maternal responses to dexmedetomidine intrathecal application during cesarean section: a meta-analysis. Medical Science Monitor. 2020; 26: e918523. 